\title{
Complex Langevin calculations in finite density QCD at large $\mu / T$ with the deformation technique
}

\author{
Keitaro Nagata* \\ KEK Theory Center, High Energy Accelerator Research Organization, \\ 1-1 Oho, Tsukuba, Ibaraki 305-0801, Japan \\ and Center of Medical Information Science, Kochi Medical School, Kochi University, \\ Kohasu, Oko-cho, Nankoku-shi, Kochi 783-8505, Japan \\ Jun Nishimura $^{\dagger}$ \\ KEK Theory Center, High Energy Accelerator Research Organization, \\ 1-1 Oho, Tsukuba, Ibaraki 305-0801, Japan \\ and Graduate University for Advanced Studies (SOKENDAI), \\ 1-1 Oho, Tsukuba, Ibaraki 305-0801, Japan \\ Shinji Shimasaki ${ }^{\ddagger}$ \\ KEK Theory Center, High Energy Accelerator Research Organization, \\ 1-1 Oho, Tsukuba, Ibaraki 305-0801, Japan \\ and Research and Education Center for Natural Sciences, Keio University, \\ Hiyoshi 4-1-1, Yokohama, Kanagawa 223-8521, Japan
}

(Received 27 September 2018; published 26 December 2018)

\begin{abstract}
It is well known that investigating QCD at finite density by standard Monte Carlo methods is extremely difficult due to the sign problem. Some years ago, the complex Langevin method with gauge cooling was shown to work at high temperature, i.e., in the deconfined phase. The same method was also applied to QCD in the so-called heavy dense limit in the whole temperature region. In this paper, we attempt to apply this method to the large $\mu / T$ regime with moderate quark mass using four-flavor staggered fermions on a $4^{3} \times 8$ lattice. While a straightforward application faces the singular-drift problem, which spoils the validity of the method, we overcome this problem by the deformation technique proposed earlier. Explicit results for the quark number density and the chiral condensate obtained in this way for $3.2 \leq \mu / T \leq 5.6$ are compared with the results for the phase-quenched model obtained by the standard rational hybrid Monte Carlo calculation. This reveals a clear difference, which is qualitatively consistent with the silver blaze phenomenon.
\end{abstract}

DOI: $10.1103 /$ PhysRevD.98.114513

\section{INTRODUCTION}

The phase diagram of QCD at finite density and temperature is speculated to have a very rich structure. This is not only interesting from theoretical viewpoints but is also relevant to the physics related to heavy-ion collision experiments and the interior structure of neutron stars. However, the speculated phase structure still remains

\footnotetext{
*k-nagata@kochi-u.ac.jp

jnishi@post.kek.jp

shimasaki.s@gmail.com
}

Published by the American Physical Society under the terms of the Creative Commons Attribution 4.0 International license. Further distribution of this work must maintain attribution to the author(s) and the published article's title, journal citation, and DOI. Funded by SCOAP ${ }^{3}$. elusive mainly because first-principle calculations based on lattice QCD are extremely difficult at finite density due to the complex fermion determinant, which causes the socalled sign problem.

As a promising solution to this problem, the complex Langevin method (CLM) [1,2] has been attracting much attention recently. In this method, based on the idea of stochastic quantization $[3,4]$, the expectation value of an observable is calculated using a stochastic process for complexified dynamical variables with the observable being extended holomorphically. Since the method does not rely on the probabilistic interpretation of the Boltzmann weight, there is a chance to overcome the sign problem completely.

However, it is known that the method yields wrong results in some cases even if the stochastic process reaches equilibrium without any problem. This issue was discussed theoretically for the first time in Refs. [5,6] by considering 
the equality between the expectation value of an observable defined by the stochastic process at each fictitious time and the expectation value of the observable with respect to a complex weight, which satisfies the Fokker-Planck equation associated with the original theory. If this equality holds, the expectation value obtained by the stochastic process in the long-time limit gives the expectation value defined by the path integral formulation of the original theory [7]. In proving this equality, a crucial role is played by the time-evolved observable, the existence of which is implicitly assumed in Refs. [5,6]. This is actually subtle and requires the condition that the probability distribution of the magnitude of the drift term in the stochastic process should fall off exponentially or faster [9]. On the other hand, if this condition is satisfied, the integration by parts used in the argument can be justified. In this sense, one may regard the above condition as a necessary and sufficient condition for justifying the CLM under such assumptions as the convergence and the ergodicity of the stochastic process. Roughly speaking, frequent appearance of large drifts during the stochastic process invalidates the CLM. The validity of this criterion has been demonstrated in simple one-variable models [9] and in semirealistic models [10].

There are actually two cases that can lead to the frequent appearance of large drifts. One is the case in which the dynamical variables make frequent excursions in the imaginary directions during the stochastic process, which is referred to as the excursion problem $[5,6]$. The other is the case in which the drift term has singularities that are frequently visited during the stochastic process, which is referred to as the singular-drift problem [8]. By avoiding these problems, one can enlarge the validity region of the CLM. For instance, the excursion problem can be solved by the gauge cooling [11], which amounts to making a complexified gauge transformation after each Langevin step in such a way that the imaginary part of the dynamical variables is minimized [12]. Theoretical justification of the gauge cooling has been given explicitly in Refs. $[9,15]$. On the other hand, Ref. [16] proposed solving the singular-drift problem by deforming the original system in such a way that the dynamical variables keep away from the singularities of the drift term. The results for the undeformed system can be obtained by extrapolating the deformation parameter to zero using the parameter region in which the criterion for justifying the CLM is satisfied. This technique was applied successfully to matrix models relevant to nonperturbative string theory [17]. For other recent developments in the CLM, see Refs. [18-32], for instance.

The gauge cooling made it possible to apply the CLM to finite density QCD in the deconfined phase $[20,33]$ and in the heavy dense limit $[11,23,34]$. In this paper, we attempt to investigate the large $\mu / T$ regime with moderate quark mass using a $4^{3} \times 8$ lattice [35]. The parameter region we are aiming at, however, is anticipated to be plagued by the singular-drift problem according to the studies of the chiral random matrix theory [40]. Indeed, we encounter this problem and use the deformation technique to solve it. By probing the probability distribution of the drift term, we determine the region of the deformation parameter in which the CLM is valid and make extrapolations to the undeformed model using the results within this region. The baryon number density and the chiral condensate thus obtained as a function of the quark chemical potential are compared with those obtained by the rational hybrid Monte Carlo (RHMC) calculation of the phase-quenched model, which is defined by omitting the phase of the fermion determinant. We observe a clear difference, which is qualitatively consistent with the so-called silver blaze phenomenon in the full model.

This paper is organized as follows. In Sec. II, we briefly review lattice QCD at finite density. In Sec. III, we explain how we apply the CLM to finite density QCD with the gauge cooling and the deformation technique. In Sec. IV, we present our results and compare them with the results for the phase-quenched model. Section V is devoted to a summary and discussions.

\section{LATTICE QCD AT FINITE DENSITY}

Our calculation is based on lattice QCD on a fourdimensional Euclidean periodic lattice defined by the partition function

$$
Z=\int \prod_{x \mu} d U_{x \mu} \operatorname{det} M(U, \mu) e^{-S_{\mathrm{g}}(U)} .
$$

The dynamical variables $U_{x \mu} \in \mathrm{SU}(3)$ are the link variables, where $x=\left(x_{1}, x_{2}, x_{3}, x_{4}\right)$ labels a site on the lattice and $\mu=1,2,3,4$ represents a direction with $\mu=1,2,3$ and $\mu=4$ being the spatial and temporal directions, respectively. We work in units that set the lattice spacing to unity and denote the number of sites in the spatial and temporal directions as $N_{\mathrm{s}}$ and $N_{\mathrm{t}}$, respectively. The gauge action $S_{\mathrm{g}}(U)$ is given by

$$
S_{\mathrm{g}}(U)=-\frac{\beta}{6} \sum_{x} \sum_{\mu<\nu} \operatorname{tr}\left(U_{x \mu \nu}+U_{x \mu \nu}^{-1}\right),
$$

where the plaquette $U_{x \mu \nu}$ is defined by $U_{x \mu \nu}=$ $U_{x \mu} U_{x+\hat{\mu}, \nu} U_{x+\hat{\nu}, \mu}^{-1} U_{x \nu}^{-1}$ with $\hat{\mu}$ being the unit vector in the $\mu$ direction.

In this work, we use the unimproved staggered fermions, for which the fermion matrix $M(U, \mu)$ in (1) is given by

$$
\begin{aligned}
M(U, \mu)_{x y}= & m \delta_{x y}+\sum_{\nu=1}^{4} \frac{1}{2} \eta_{\nu}(x)\left(e^{\mu \delta_{\nu 4}} U_{x \nu} \delta_{x+\hat{\nu}, y}\right. \\
& \left.-e^{-\mu \delta_{\nu 4}} U_{x-\hat{\nu}, \nu}^{-1} \delta_{x-\hat{\nu}, y}\right),
\end{aligned}
$$

where $\eta_{\nu}(x)=(-1)^{x_{1}+\cdots+x_{\nu-1}}$. This represents four flavors of quarks with the degenerate quark mass $m$ and the quark 
chemical potential $\mu$. The quark field obeys the periodic/ antiperiodic boundary conditions in the spatial/temporal directions, respectively. Note that the fermion matrix $M(U, \mu)$ satisfies $\epsilon_{x} M(U, \mu)_{x y} \epsilon_{y}=M\left(U,-\mu^{*}\right)_{y x}^{*}$ with $\epsilon_{x}=$ $(-1)^{x_{1}+x_{2}+x_{3}+x_{4}}$ playing the role of $\gamma_{5}$. Hence, for nonzero real $\mu$, the fermion determinant $\operatorname{det} M(U, \mu)$ becomes complex in general, causing the sign problem, which we overcome by the CLM explained in the next section.

The observables we consider in this paper are the baryon number density

$$
\langle n\rangle=\frac{1}{3 N_{\mathrm{V}}} \frac{\partial}{\partial \mu} \log Z
$$

and the chiral condensate

$$
\langle\Sigma\rangle=\frac{1}{N_{\mathrm{V}}} \frac{\partial}{\partial m} \log Z,
$$

where $N_{\mathrm{V}}=N_{\mathrm{s}}^{3} N_{\mathrm{t}}$. We use the standard noisy estimator to calculate these quantities. Details related to this method are given in Appendix A.

\section{COMPLEX LANGEVIN METHOD FOR FINITE DENSITY QCD}

In this section, we explain how we apply the CLM [1,2] to lattice QCD at finite density. First, we extend the link variables $U_{x \mu} \in \mathrm{SU}(3)$ to the complexified link variables $\mathcal{U}_{x \mu} \in \operatorname{SL}(3, \mathbb{C})$ and consider their fictitious time evolution based on the complex Langevin equation, which is given in its discrete form by

$$
\begin{aligned}
& \mathcal{U}_{x \mu}(t+\epsilon) \\
& =\exp \left(i \sum_{a=1}^{8} \lambda_{a}\left[-\epsilon v_{a x \mu}(\mathcal{U}(t))+\sqrt{\epsilon} \eta_{a x \mu}(t)\right]\right) \mathcal{U}_{x \mu}(t),
\end{aligned}
$$

where $t$ represents the discretized Langevin time with the step size $\epsilon$. We have introduced the generators $\lambda_{a}(a=1, \ldots, 8)$ of the $\mathrm{SU}(3)$ algebra normalized as $\operatorname{tr}\left(\lambda_{a} \lambda_{b}\right)=\delta_{a b}$ and the real Gaussian noise $\eta_{a x \mu}(t)$ normalized as $\left\langle\eta_{a x \mu}(t) \eta_{b y \nu}\left(t^{\prime}\right)\right\rangle_{\eta}=2 \delta_{a b} \delta_{x y} \delta_{\mu \nu} \delta_{t t^{\prime}}$, where $\langle\cdots\rangle_{\eta}$ represents an average over $\eta$. The drift term $v_{a x \mu}(\mathcal{U})$ in (6) is defined by analytic continuation of the one defined for $U_{x \mu} \in \mathrm{SU}(3)$ as

$$
v_{a x \mu}(U)=D_{a x \mu} S(U) \equiv \lim _{\varepsilon \rightarrow 0} \frac{S\left(e^{i \varepsilon \lambda_{a}} U_{x \mu}\right)-S\left(U_{x \mu}\right)}{\varepsilon},
$$

where $S(U)=S_{\mathrm{g}}(U)-\log \operatorname{det} M(U, \mu)$.

To calculate the vacuum expectation value (VEV) of a gauge-invariant observable $O(U)$, we define $O(\mathcal{U})$ by analytic continuation and its expectation value

$$
\Phi(t) \equiv\langle O(\mathcal{U}(t))\rangle_{\eta}
$$

in the CLM. Then, under certain conditions, one can prove that

$$
\lim _{t \rightarrow \infty} \lim _{\epsilon \rightarrow 0} \Phi(t)=\frac{1}{Z} \int \prod_{x \mu} d U_{x \mu} O(U) e^{-S}
$$

which implies that the left-hand side gives the VEV of $O(U)$ in the original theory.

As is mentioned in the Introduction, the proof of (9) was first given in Refs. [5,6] and was refined later by Ref. [9], which showed that the necessary and sufficient condition for (9) to hold is that the probability distribution of the magnitude of the drift term should fall off exponentially or faster. In this work, we define the magnitude of the drift term as

$$
u=\left(\frac{1}{8 N_{\mathrm{V}}} \sum_{x \mu} \sum_{a=1}^{8}\left|v_{a x \mu}(\mathcal{U})\right|^{2}\right)^{\frac{1}{2}}
$$

and probe its probability distribution in order to see whether the CLM is valid or not at each set of parameters. The validity of this criterion is tested not only in simple onevariable models [9] but also in semirealistic many-variable systems [10].

It is known that the slow fall-off of the drift distribution, which invalidates (9), is caused either by the excursion problem or by the singular-drift problem. In finite density QCD, the former problem occurs when the complexified link variables become far from unitary, while the latter problem occurs when the fermion matrix (3) has many eigenvalues close to zero for the complexified link variables.

To solve the excursion problem, one can use the gauge cooling, which amounts to making a complexified gauge transformation after each Langevin step in such a way that the complexified link variables come closer to a unitary configuration [11]. The gauge transformation can be determined by minimizing the unitarity norm

$\mathcal{N}_{u}=\frac{1}{4 N_{\mathrm{V}}} \sum_{x \mu} \operatorname{tr}\left[\left(\mathcal{U}_{x \mu}\right)^{\dagger} \mathcal{U}_{x \mu}+\left(\mathcal{U}_{x \mu}^{-1}\right)^{\dagger} \mathcal{U}_{x \mu}^{-1}-2 \times \mathbf{1}_{3 \times 3}\right]$,

which measures how far the link variables are from a unitary configuration. It has been shown explicitly $[9,15]$ that this additional procedure does not affect the argument for justifying the CLM. The gauge cooling played a crucial role in enabling the application of the CLM to finite density QCD in the deconfined phase [33] and in the heavy dense limit $[11,23,34]$.

At large $\mu / T$ with moderate quark mass, the singulardrift problem occurs on top of the excursion problem. To solve that problem, we use the deformation technique [16], which was applied successfully to matrix models relevant to superstring theory [17]. 
In the case at hand, we introduce a deformation parameter $\alpha \in \mathbb{R}$ in the fermion matrix (3) as

$$
M(U, \mu)_{x y} \rightarrow M(U, \mu)_{x y}+i \alpha \eta_{4}(x) \delta_{x y} .
$$

This deformation may be regarded as adding an imaginary chemical potential in the continuum theory. Strictly speaking, the extra term corresponds to adding a term $i \alpha \bar{\psi}(x)\left(\gamma_{4} \otimes \gamma_{4}\right) \psi(x)$ in the Lagrangian density of the continuum theory, where the first $\gamma_{4}$ acts on the spinor indices and the second $\gamma_{4}$ acts on the flavor indices.

For $\alpha$ large enough, the eigenvalue distribution of the fermion matrix develops a gap near the real axis, which enables us to avoid the singular-drift problem. When the singular-drift problem occurs, the unitarity norm (11) becomes large, and it sometimes becomes uncontrollable. This problem is cured when the singular-drift problem is avoided by sufficiently large $\alpha$.

We probe the drift distribution at each $\alpha$ and determine the range of $\alpha$ for which the obtained results are reliable. Extrapolating the results within this range of $\alpha$ to $\alpha=0$, we obtain the results for the original theory. Considering the symmetry of the deformed theory under $\alpha \leftrightarrow-\alpha$, we choose the fitting function to be a linear function of $\alpha^{2}$.

\section{RESULTS}

In this section, we show our results for finite density QCD obtained by the CLM as explained in the previous section. We use a $4^{3} \times 8$ lattice with the gauge coupling $\beta=5.7$ and the quark mass $m=0.05$. The quark chemical potential $\mu$ is taken to be $0.4 \leq \mu \leq 0.7$, which implies that the physical $\mu / T$ ranges from 3.2 to 5.6. The Langevin process (6) is performed for the total Langevin time 50-150 with a fixed step size $\epsilon=10^{-4}$. We present results for the baryon number density (4) and the chiral condensate (5), which are compared with those for the phase-quenched model obtained by the standard RHMC calculation.

First, we check the validity of the CLM by probing the probability distribution of the drift term, which is shown in Fig. 1 for $\mu=0.7$ with various $\alpha$. We find that the probability distribution falls off exponentially or faster for $\alpha \geq 0.4$, while a power-law tail develops for $\alpha=0.2$, 0.3. This implies that the CLM is valid for $\alpha \geq 0.4$ at $\mu=0.7$.

The power-law tail of the probability distribution for $\alpha \lesssim$ 0.3 is actually due to the singular-drift problem caused by near-zero eigenvalues of the fermion matrix, as one can see from Fig. 2. Indeed, there are many eigenvalues distributed around the origin for $\alpha=0.2$, which is not the case for $\alpha=0.4$, owing to the gap developing along the real axis.

In Fig. 3, we plot the baryon number density $\langle n\rangle$ (top) and the chiral condensate $\langle\Sigma\rangle$ (bottom) obtained by the CLM against $\alpha^{2}$ for $\mu=0.7$. Note that the data points for $\alpha \lesssim 0.3$ should be discarded since the CLM is not valid there. We find that $\langle n\rangle$ drops to zero for $\alpha \geq 0.6$ and that

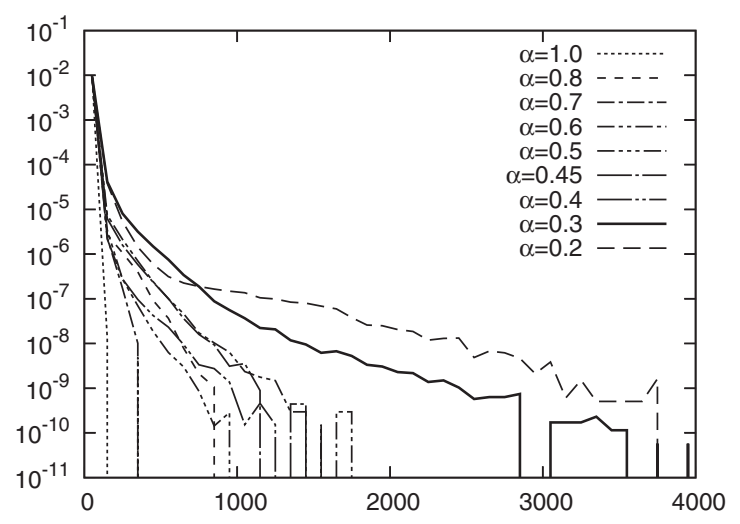

FIG. 1. The probability distribution of $u$, the magnitude of the drift term defined by (10), is shown in a semilog plot for $\mu=0.7$ with various $\alpha$.

$\langle\Sigma\rangle$ changes its behavior at $\alpha \sim 0.6$. These observations suggest the existence of a phase transition at $\alpha \sim 0.6$. Thus, we are led to use only the data points for $\alpha=0.4,0.45,0.5$ for the extrapolation to $\alpha=0$ for $\mu=0.7$. These are shown in Fig. 4 by circles, which can be fitted to a straight line. In the same figure, we also plot the reliable data points obtained for other values of $\mu$ together with the linear extrapolation using $\alpha=0.1,0.2,0.3$ for $\mu=0.4$ and $\alpha=0.2,0.3,0.4$ for $\mu=0.5,0.6$.

When we performed complex Langevin simulations without deformation, the history of observables typically
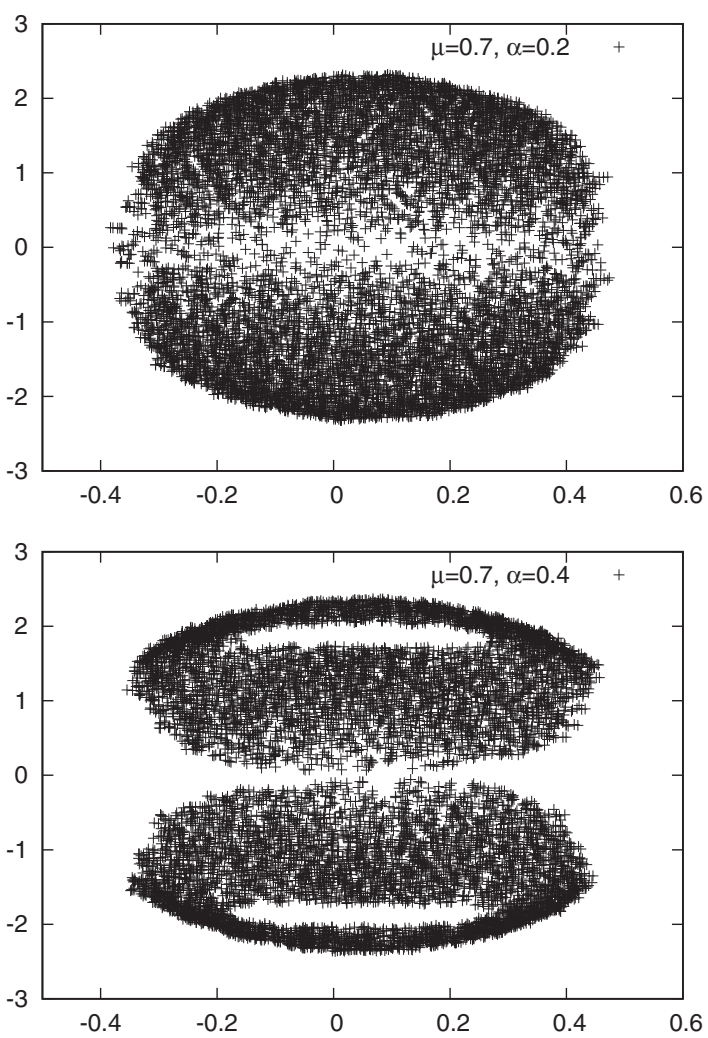

FIG. 2. The eigenvalue distribution of the fermion matrix is shown for $\mu=0.7$ with $\alpha=0.2$ (top) and $\alpha=0.4$ (bottom). 

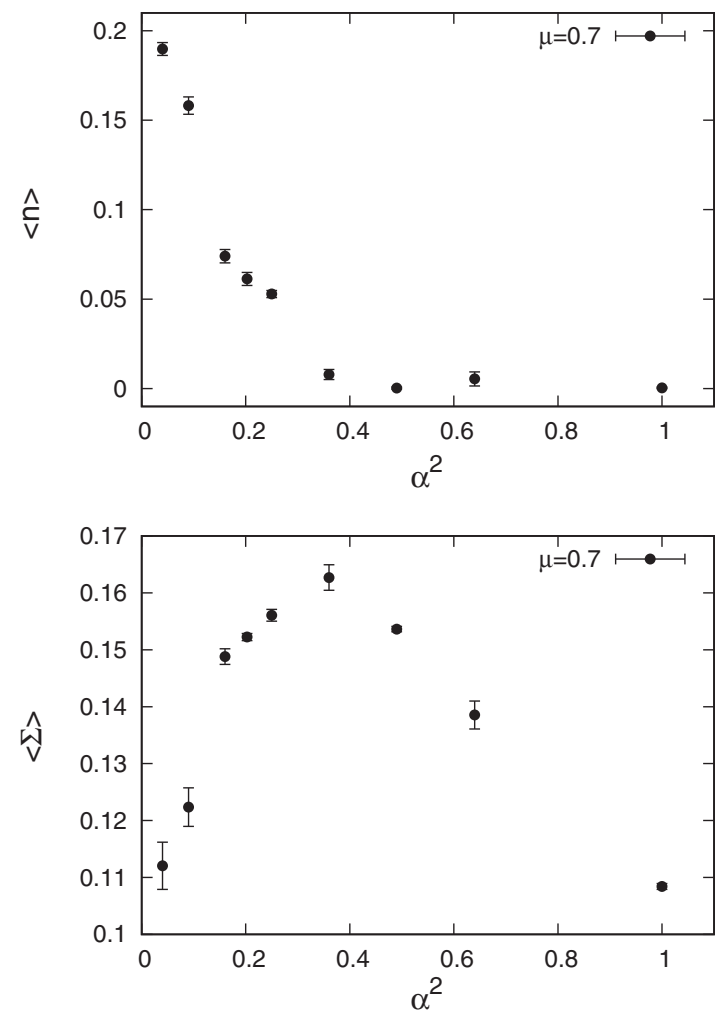

FIG. 3. The baryon number density (top) and the chiral condensate (bottom) obtained by the CLM are plotted against $\alpha^{2}$ for $\mu=0.7$.

shows occasional spikes, which makes it difficult to reduce the statistical error within a reasonable computing time [41]. This problem does not occur in all the cases investigated here with the deformation.

In Fig. 5, the extrapolated values for the baryon number density (top) and the chiral condensate (bottom) are plotted against $\mu$ by circles. In the same figure, we also plot by squares the results for the phase-quenched model obtained by the standard RHMC calculation, which reveals a clear difference. The results of the CLM show that the baryon number density is almost zero for $\mu \lesssim 0.6$ and has a sharp increase within $0.6 \lesssim \mu \lesssim 0.7$. Correspondingly, the chiral condensate is almost constant for $\mu \lesssim 0.6$ and starts to decrease rapidly within $0.6 \lesssim \mu \lesssim 0.7$. While certain systematic errors due to the $\alpha \rightarrow 0$ extrapolation are considered to exist, the rapid change within $0.6 \lesssim \mu \lesssim 0.7$ should be robust, judging from the qualitative difference of the $\alpha$ dependence for $\mu=0.6$ and $\mu=0.7$ seen in Fig. 4. The results for the phase-quenched model, on the other hand, show a milder $\mu$ dependence. The onset of the baryon number density occurs around $\mu \sim 0.4$, where the chiral condensate starts to decrease.

Note that the value of $\beta$ is chosen to be large $(\beta=5.7)$ in order to avoid the excursion problem that occurs at smaller $\beta$ similarly to the situation found in Ref. [20]. Because of this, the corresponding lattice spacing is well below $0.1 \mathrm{fm}$ according to a crude extrapolation from the data obtained in
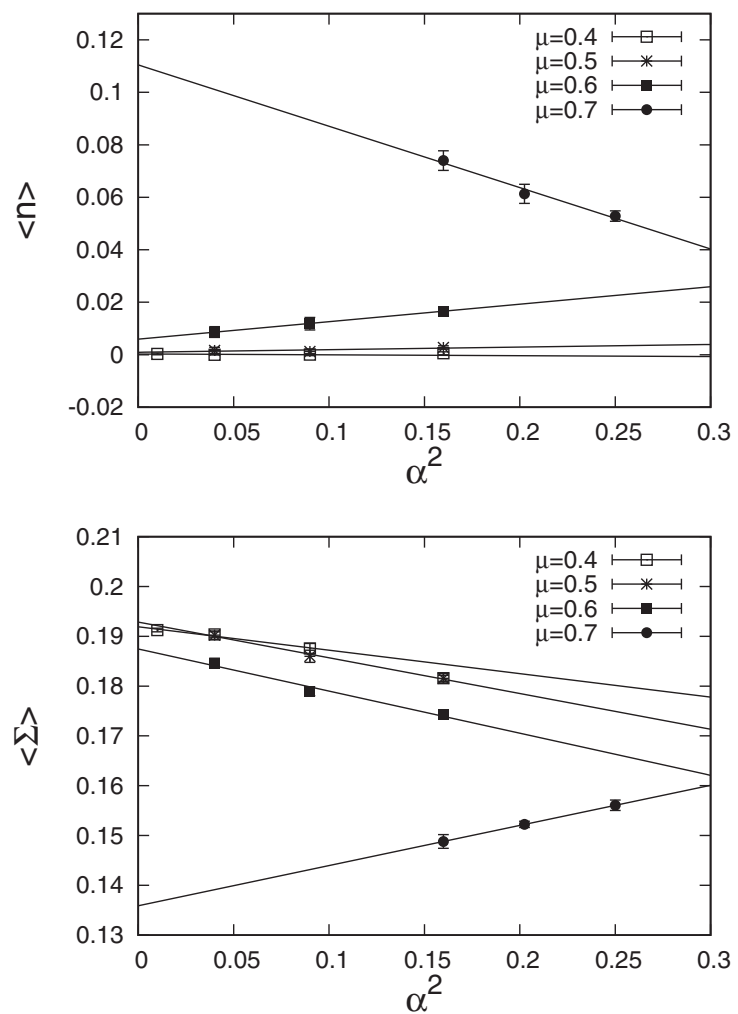

FIG. 4. The baryon number density (top) and the chiral condensate (bottom) obtained by the CLM are plotted against $\alpha^{2}$ for $\mu=0.4,0.5,0.6,0.7$. We present only the data points that are reliable in light of the drift distribution. The straight lines represent linear extrapolations to $\alpha=0$ with respect to $\alpha^{2}$.

Ref. [20]. Considering that our lattice is $4^{3} \times 8$, this implies that the temperature is quite high, whereas the physical volume of the spatial lattice is much smaller than the QCD scale. In fact, despite the high temperature, the Polyakov line vanishes for $\mu \leq 0.6$, as we show in Appendix B. This can be understood as a consequence of the finite spatial volume effects since the increase of free energy for having one quark in such a small spatial region is much larger than that in the infinite volume at the same temperature. In other words, the temperature is actually "low" compared with the scale of the spatial directions due to the chosen aspect ratio. It is therefore not so surprising that the observables behave more like those at low temperature in a usual setup.

In full QCD at zero temperature in the infinite volume limit, physical observables are independent of $\mu$ up to $\mu \sim$ $m_{\mathrm{N}} / 3$ with $m_{\mathrm{N}}$ being the nucleon mass. On the other hand, in the case of the phase-quenched model, physical observables are independent of $\mu$ up to $\mu \sim m_{\pi} / 2\left(<m_{\mathrm{N}} / 3\right)$ with $m_{\pi}$ being the pion mass. The $\mu$ independence of full QCD within the region $m_{\pi} / 2<\mu<m_{\mathrm{N}} / 3$, which is commonly referred to as the "silver blaze phenomenon" in the literature, is expected to occur due to the effect of the phase of the complex fermion determinant. Our results are qualitatively consistent with this expectation. 

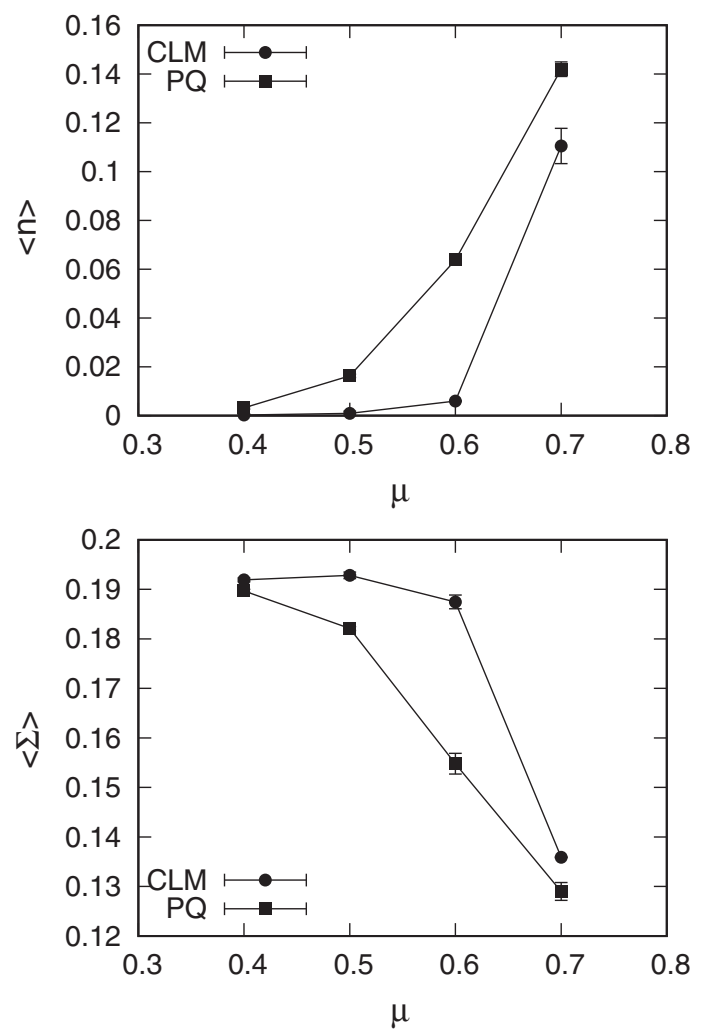

FIG. 5. The extrapolated values of the baryon number density (top) and the chiral condensate (bottom) obtained from Fig. 4 are plotted against $\mu$. The solid lines are drawn to guide the eye.

\section{SUMMARY AND DISCUSSIONS}

We have made an attempt to extend the success of the CLM in investigating finite density QCD in the deconfined phase or in the heavy dense limit to the large $\mu / T$ regime with moderate quark mass. In this exploratory work, we use a $4^{3} \times 8$ lattice with four-flavor staggered fermions and calculate the baryon number density and the chiral condensate as a function of the quark chemical potential. The reliability of the obtained results is judged by the probability distribution of the magnitude of the drift term. As in the previous work, the excursion problem is avoided by the gauge cooling. In addition to this, the singular-drift problem has to be overcome in the parameter regime we explore. The deformation technique, which was shown to be useful in the case of matrix models for superstring theory, turns out to be useful also in the present case. By probing the probability distribution of the magnitude of the drift term, we find that the singular-drift problem can be cured and reliable data can be obtained unless the deformation parameter is too small. The results for the original theory are obtained by extrapolation using only the reliable data. Thus, we are able to obtain explicit results in the region $3.2 \leq \mu / T \leq 5.6$ with moderate quark mass.

By comparing the results of the CLM with those obtained by the RHMC calculations in the phase-quenched model, we observe that the onset of the baryon number density in the full model occurs at larger $\mu$ than in the phase-quenched model, which is qualitatively consistent with the silver blaze phenomenon, which occurs at zero temperature in the infinite volume. To confirm this phenomenon, we clearly need to increase the lattice size. We have already started simulations on a $8^{3} \times 16$ lattice [42] and found that the CLM actually gives correct results even in the region of relatively large chemical potential without the deformation technique. Preliminary results for the baryon number density and the chiral condensate show a rapid change twice as we increase the chemical potential, which may be interpreted as the phase transitions to the nuclear matter and to the quark matter. We hope to report on these results in the forthcoming publication.

\section{ACKNOWLEDGMENTS}

The authors would like to thank Y. Ito, T. Kaneko, H. Matsufuru, K. Moritake, A. Tsuchiya, and S. Tsutsui for valuable discussions. Computations were carried out on Cray XC40 at YITP in Kyoto University, SX-ACE at CMC and RCNP in Osaka University and PC clusters at KEK. K. N. and J. N. were supported in part by Grant-inAid for Scientific Research (Grants No. 26800154 and No. 16H03988, respectively) from Japan Society for the Promotion of Science. S. S. was supported by the MEXTSupported Program for the Strategic Research Foundation at Private Universities "Topological Science" (Grant No. S1511006).

\section{APPENDIX A: DETAILS OF THE NOISY ESTIMATOR}

To calculate the drift term

$$
v_{a x \mu}^{(\mathrm{f})}=-\operatorname{tr}\left(M^{-1} D_{a x \mu} M\right)
$$

obtained from the fermion determinant, we use the standard noisy estimator. In this section, we present the details of how we use this method in our calculation.

The idea is to replace the trace in (A1) by

$$
v_{a x \mu}^{(\mathrm{f})}=-\varphi^{*} M^{-1} D_{a x \mu} M \varphi=-\psi^{*} D_{a x \mu} M \varphi,
$$

where $\varphi$ is a random complex vector generated with the normalized Gaussian distribution. The other complex vector $\psi=\left(M^{\dagger}\right)^{-1} \varphi$ in (A2) can be calculated from $\varphi$ as $\psi=M \chi$, where $\chi$ is obtained by solving $M^{\dagger} M \chi=\varphi$ using the conjugate gradient method.

The above procedure is exact if we take an average over infinitely many $\varphi$ generated randomly. In practice, we generate the random vector only once at each Langevin step and estimate the trace using it. The use of this approximation does not yield any systematic errors in the CLM in the step size $\epsilon \rightarrow 0$ limit since the associated Fokker-Planck 
equation remains the same [43]. When we evaluate the trace involved in the observables (4) and (5), we take an average over $20 \varphi$ 's generated randomly.

See Refs. [37-39] for a more sophisticated way to implement the noisy estimator, which ensures that the drift term becomes real for unitary link variables at $\mu=0$.

\section{APPENDIX B: THE RESULTS FOR THE POLYAKOV LINE}

In this Appendix, we present our results for the Polyakov line defined by

$$
P=\frac{1}{3 N_{\mathrm{s}}^{3}} \sum_{\vec{x}} \operatorname{tr}\left(U_{(\vec{x}, 0) 4} U_{(\vec{x}, 1) 4} \cdots U_{\left(\vec{x}, N_{\mathrm{t}}-1\right) 4}\right) .
$$

Figure 6 shows the expectation value $\langle P\rangle$ plotted against $\alpha^{2}$. We plot only the reliable data from the viewpoint of our criterion based on the drift distribution. For $\mu=0.4$ and $\mu=0.7$, we can fit the data to a straight line. For $\mu=0.5$ and $\mu=0.6$, we find that the data are close to zero for sufficiently small $\alpha$.

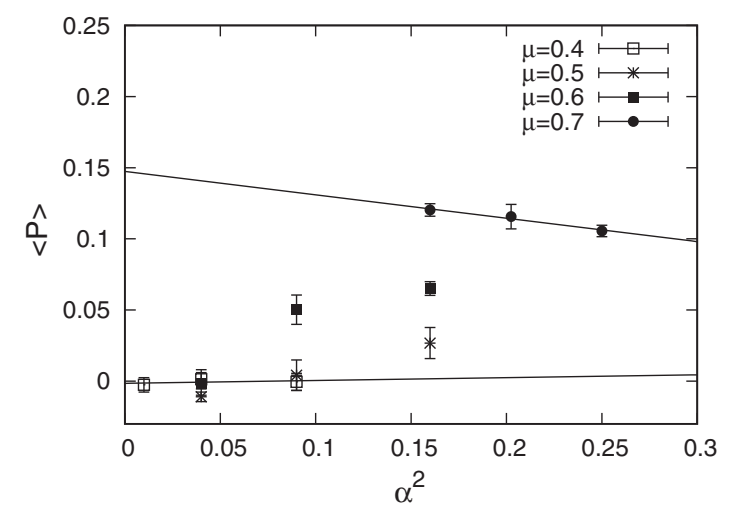

FIG. 6. The Polyakov line obtained by the CLM is plotted against $\alpha^{2}$ for $\mu=0.4,0.5,0.6,0.7$. We present only the data points that are reliable in the light of the drift distribution. The straight lines represent linear extrapolations to $\alpha=0$ with respect to $\alpha^{2}$ for $\mu=0.4$ and $\mu=0.7$.

In the phase-quenched model, the expectation value $\langle P\rangle$ is obtained as $0.594(1), 0.749(2), 0.121(3), 0.146(4)$ for $\mu=0.4,0.5,0.6,0.7$, respectively.
[1] G. Parisi, Phys. Lett. 131B, 393 (1983).

[2] J. R. Klauder, Phys. Rev. A 29, 2036 (1984).

[3] G. Parisi and Y.-s. Wu, Sci. Sin. 24, 483 (1981).

[4] P. H. Damgaard and H. Huffel, Phys. Rep. 152, 227 (1987).

[5] G. Aarts, E. Seiler, and I.-O. Stamatescu, Phys. Rev. D 81, 054508 (2010).

[6] G. Aarts, F. A. James, E. Seiler, and I.-O. Stamatescu, Eur. Phys. J. C 71, 1756 (2011).

[7] For this to be true, it has long been considered that all the eigenvalues of the Fokker-Planck Hamiltonian should have a positive real part. However, it was argued in Ref. [8] with explicit examples that this condition is automatically satisfied if the aforementioned equality holds.

[8] J. Nishimura and S. Shimasaki, Phys. Rev. D 92, 011501 (2015).

[9] K. Nagata, J. Nishimura, and S. Shimasaki, Phys. Rev. D 94, 114515 (2016).

[10] K. Nagata, J. Nishimura, and S. Shimasaki, J. High Energy Phys. 05 (2018) 004.

[11] E. Seiler, D. Sexty, and I.-O. Stamatescu, Phys. Lett. B 723, 213 (2013).

[12] This technique was also used to solve the singular-drift problem in the chiral random matrix theory [13]. See Ref. [14], however, for a case in which it does not work.

[13] K. Nagata, J. Nishimura, and S. Shimasaki, J. High Energy Phys. 07 (2016) 073.

[14] J. Bloch, J. Glesaaen, J. J. M. Verbaarschot, and S. Zafeiropoulos, J. High Energy Phys. 03 (2018) 015.

[15] K. Nagata, J. Nishimura, and S. Shimasaki, Prog. Theor. Exp. Phys. 2016, 013B01 (2016).
[16] Y. Ito and J. Nishimura, J. High Energy Phys. 12 (2016) 009.

[17] K. N. Anagnostopoulos, T. Azuma, Y. Ito, J. Nishimura, and S. K. Papadoudis, J. High Energy Phys. 02 (2018) 151.

[18] H. Makino, H. Suzuki, and D. Takeda, Phys. Rev. D 92, 085020 (2015).

[19] S. Tsutsui and T. M. Doi, Phys. Rev. D 94, 074009 (2016).

[20] Z. Fodor, S. D. Katz, D. Sexty, and C. Török, Phys. Rev. D 92, 094516 (2015).

[21] T. Hayata, Y. Hidaka, and Y. Tanizaki, Nucl. Phys. B911, 94 (2016).

[22] T. Ichihara, K. Nagata, and K. Kashiwa, Phys. Rev. D 93, 094511 (2016).

[23] G. Aarts, F. Attanasio, B. Jäger, and D. Sexty, J. High Energy Phys. 09 (2016) 087.

[24] Y. Abe and K. Fukushima, Phys. Rev. D 94, 094506 (2016).

[25] G. Aarts, F. Attanasio, B. Jäger, and D. Sexty, Acta Phys. Pol. B Proc. Suppl. 9, 621 (2016).

[26] L. L. Salcedo, Phys. Rev. D 94, 114505 (2016).

[27] J. Bloch, Phys. Rev. D 95, 054509 (2017).

[28] G. Aarts, E. Seiler, D. Sexty, and I.-O. Stamatescu, J. High Energy Phys. 05 (2017) 044.

[29] J. Nishimura and S. Shimasaki, J. High Energy Phys. 06 (2017) 023.

[30] T. M. Doi and S. Tsutsui, Phys. Rev. D 96, 094511 (2017).

[31] H. Fujii, S. Kamata, and Y. Kikukawa, arXiv:1710.08524.

[32] P. Basu, K. Jaswin, and A. Joseph, Phys. Rev. D 98, 034501 (2018).

[33] D. Sexty, Phys. Lett. B 729, 108 (2014). 
[34] G. Aarts, L. Bongiovanni, E. Seiler, D. Sexty, and I.-O. Stamatescu, Eur. Phys. J. A 49, 89 (2013).

[35] Preliminary results are presented in Lattice 2017 [36]. See also Refs. [37-39] for related work.

[36] K. Nagata, J. Nishimura, and S. Shimasaki, EPJ Web Conf. 175, 07017 (2018).

[37] D. K. Sinclair and J. B. Kogut, Proc. Sci. LATTICE2015 (2016) 153.

[38] D. K. Sinclair and J. B. Kogut, Proc. Sci. LATTICE2016 (2016) 026.
[39] D. K. Sinclair and J. B. Kogut, EPJ Web Conf. 175, 07031 (2018).

[40] A. Mollgaard and K. Splittorff, Phys. Rev. D 88, 116007 (2013).

[41] K. Nagata, H. Matsufuru, J. Nishimura, and S. Shimasaki, Proc. Sci. LATTICE2016 (2016) 067.

[42] Y. Ito, H. Matsufuru, K. Moritake, J. Nishimura, S. Shimasaki, A. Tsuchiya, and S. Tsutsui (to be published).

[43] G. G. Batrouni, G. R. Katz, A. S. Kronfeld, G. P. Lepage, B. Svetitsky, and K. G. Wilson, Phys. Rev. D 32, 2736 (1985). 